\title{
El ciclo hidrosocial en la ciudad de Torrevieja: retos y nuevas tendencias ${ }^{1}$
}

\author{
Ana Arahuetes², Rubén Villar ${ }^{3}$ y María Hernández ${ }^{4}$
}

\begin{abstract}
RESUMEN
La costa mediterránea española ha necesitado introducir, durante la última década, nuevas fuentes de suministro hídrico con el fin de satisfacer la creciente demanda. Partiendo de esta situación, este estudio tiene entre sus objetivos evidenciar las características del ciclo hidrosocial en la ciudad de Torrevieja y, a partir de este análisis, poner de manifiesto las medidas que se han adoptado y han permitido asegurar el suministro en un contexto de déficit hídrico intensificado por la expansión urbanística y las demandas agrícolas. Para la consecución de estos objetivos se analizan las relaciones entre actores, procesos metabólicos y origen de los recursos hídricos que conforman este ciclo hidrosocial, desde la perspectiva teórica de la Ecología Política Urbana. La utilización de esta metodología pone de manifiesto que el uso de recursos no convencionales ha aliviado la demanda agrícola mediante el agua depurada, pero ha generado nuevas problemáticas al incorporar el agua desalada.
\end{abstract}

Palabras clave: Ciclo hidrosocial, recursos hídricos, reutilización, desalinización, España.

\begin{abstract}
The Spanish Mediterranean coast has needed to introduce, over the last decade, new sources of water supply in order to meet growing demand. Based on this situation, one of this study's objectives is to demonstrate the characteristics of the hydrosocial cycle in the city of Torrevieja and from this analysis, highlighting the measures that have been taken and have ensured the supply in a context of deficit water intensified by urban sprawl and large agricultural demands. To achieve these objectives the relations between actors, metabolic processes and origin of water resources that form this hydrosocial cycle, from the theoretical perspective of Urban Political Ecology, are analyzed. The use of this methodology shows that the use of non-conventional resources has eased agricultural demand through reclaimed water, but has created new problems by incorporating desalinated water.
\end{abstract}

Key words: Hydrosocial cycle, water resources, reuse, desalination, Spain. 
La necesidad de ampliar las fuentes de abastecimiento para satisfacer las crecientes demandas de agua potable durante los crecimientos urbanísticos y poblacionales de las ciudades ha quedado patente a lo largo de los años (March et al., 2015a; March, 2013; Melosi, 2000). Como pone de manifiesto Ridolfi (2014), la complejidad de estos desafíos hace necesaria la incorporación de nuevas perspectivas teóricas y metodológicas para analizar, desde la Geografía, los flujos de agua urbanos. Ante este reto, la Ecología Política Urbana propone el enfoque epistemológico del metabolismo urbano, que entiende las ciudades como un producto híbrido socio-natural que es alimentado por la movilización de diversos flujos naturales, entre los que se encuentra el agua. También atiende a los factores políticos y socioeconómicos que motivan la circulación de los flujos hídricos, por lo que se propone abandonar el antiguo paradigma del ciclo hidrológico para incorporar el de ciclo hidrosocial. De este modo, se inserta la influencia antrópica que convierte este ciclo en un proceso híbrido socio-natural donde agua y sociedad se influyen mutuamente en el espacio y a lo largo del tiempo (Swyngedouw, 2004, 2009; Linton y Budds, 2014; Schmidt, 2014).

En España, el carácter político del agua ha sido estudiado a raíz de la hegemonía del paradigma hidráulico, constituido a lo largo del S.XX y especialmente durante el régimen de Franco (Swyngedouw, 1999), lo que motivó un gran desarrollo de las infraestructuras hidráulicas para asegurar los flujos de agua necesarios para incrementar la superficie de regadío y posibilitar el desarrollo urbanístico y turístico a lo largo de la costa mediterránea desde la década de 1960. Esta configuración del ciclo hidrosocial nacional fue posible gracias a los nuevos acuerdos geopolíticos que aseguraron los flujos de capitales para la construcción de esas infraestructuras y a los intereses de las élites que apoyaron al régimen de Franco y que se vieron beneficiadas por la particular configuración de las políticas hídricas (Swyngedouw, 2007, 2014, 2015).

Este contexto permite entender las bases sociales, económicas y políticas que han cimentado el paradigma hidráulico en España, posibilitando el aumento de la demanda de agua en territorios con un déficit hídrico estructural y sequías recurrentes como el sureste de España, donde se ubica Torrevieja. El estudio del balance hídrico de la zona denota que la demanda se encuentra muy por encima de los recursos disponibles (Morales et al., 2005). En concreto, para el año 2003 en la comarca de la Vega Baja del Segura, donde se inserta Torrevieja, la situación entre recursos disponibles (268 hm³/año) y las demandas generadas para todos los usos (335 hm²/año) presentaba un déficit hídrico de $67 \mathrm{hm}^{3} /$ año (Generalitat, 2003). A escala regional, los datos proporcionados por la Demarcación Hidrográfica del Segura (DHS), organismo de cuenca que gestiona el agua en la mayor parte de la provincia de Murcia y el sur de Alicante, presentaban, por un lado, una variabilidad de recursos disponibles que oscilaba entre 1.555 y $1.923 \mathrm{hm} 3 /$ año dependiendo de las variables naturales y de la posibilidad de contar con los máximos caudales del trasvase Tajo-Segura. Por otro, las demandas para el horizonte 2015 se situaban en 1.847 hm³/año, y las proyecciones señalan un ligero incremento de los usos urbanos e industriales hasta los $1.903 \mathrm{hm} 3 /$ año para 2027 (Demarcación Hidrográfica del Segura, 2014). Asimismo, se espera una disminución a medio y largo plazo de los recursos disponibles como consecuencia directa del cambio climático (Giorgi y Lionello, 2008). Según los datos manejados por la Demarcación Hidrográfica del Segura, esta reducción de las aportaciones naturales se situaría en torno al 11\% para el año 2027; aunque algunos autores afirman que no se han recabado datos suficientes que avalen la disminución de precipitaciones en la región geográfica del Sureste Ibérico, caracterizada por poseer un clima donde los fuertes contrastes pluviométricos son la norma (Gil, 2009). 
La falta de recursos hídricos provenientes de fuentes convencionales, la amenaza al suministro que suponen las previsiones asociadas al cambio climático y la necesidad de resolver los conflictos interregionales asociados al trasvase Tajo-Segura y al trasvase del Ebro, propuesto en el Plan Hidrológico Nacional (PHN) de 2001 y derogado posteriormente, evidencian la necesidad de un cambio en las políticas hidráulicas (Saurí y del Moral, 2001). La gran oposición que en algunas regiones tuvo el PHN fue posiblemente el desencadenante que posibilitó la creación del movimiento por una Nueva Cultura del Agua (Ayala-Carcedo, 1999; Martínez, 1997). Corriente que surge a principios de los años noventa como respuesta ante una gestión del agua centralizada basada en un aumento de la oferta a través de grandes infraestructuras hidráulicas y trasvases, proponiendo un nuevo enfoque basado en la gestión de la demanda y en buscar alternativas sostenibles desde el punto de vista ambiental, social y económicamente a los recursos hídricos convencionales. Es en este marco donde se inserta la apuesta por los recursos no convencionales, constituidos básicamente por la desalación y la reutilización de aguas residuales depuradas.

La desalinización fue entendida como la alternativa de suministro más adecuada para equilibrar los balances hídricos en las cuencas deficitarias, ya que, por un lado, acabaría con los conflictos interregionales y las tensiones sociales generadas en torno a la construcción de trasvases $y$, por otro, suponía una medida eficaz ante la escasez de recursos en las regiones mediterráneas, acentuada en periodos de sequía, al disponer de un recurso (el agua de mar) independiente de las condiciones climáticas; asegurando de este modo las demandas hídricas en auge. El gobierno del PSOE que accedió al gobierno en 2004, propuso un desarrollo sin precedentes de la desalación a través del Programa de Actuaciones para la Gestión y Utilización del Agua (Programa AGUA) (Olcina y Moltó, 2010). Este programa surge como resultado de la derogación del trasvase del Ebro y la modificación del Plan Hidrológico Nacional de 2001 (Rico, 2010; March et al., 2014), y se proyectó en base a las tendencias de crecimiento inmobiliario y aumento de las demandas hídricas de comienzos de la década del 2000. La apuesta por este recurso no convencional no ha estado exenta de un fuerte debate que se prolonga hasta la actualidad y donde sus detractores argumentan unos costes económicos y ambientales muy elevados (Rico, 2010). Los años de bonanza económica impulsaron estas grandes y costosas infraestructuras hídricas en toda España, con una inversión total estimada alrededor de los 4 billones de euros que convirtieron a España en el país occidental con mayor capacidad de desalación (Swyngedouw, 2013, 2014). Sin embargo, no se han cumplido las expectativas en cuanto a su utilización, lo que ha puesto de manifiesto el sobredimensionamiento de la capacidad de producción de estas plantas. El estallido de la burbuja inmobiliaria y el elevado coste del agua desalada, ha determinado que los usuarios prefieran acceder a otras fuentes de suministro más baratas (Rico, 2010; March et al., 2014); siendo posible actualmente cubrir la demanda de agua recurriendo principalmente a los recursos convencionales y empleándose la desalinización solo en casos de extrema sequía o emergencia; convirtiéndose en un recurso estratégico (Arahuetes, 2015).

Algunos autores (Swyngedouw, 2013, 2014; March, 2015) conciben este desarrollo de la desalinización en España como un nuevo arreglo socio-natural al problema del agua, que extiende el ciclo hidrosocial hasta el mar, que se presenta como la solución a los problemas de escasez. De esta manera, el Estado perpetúa el modelo de negocios del paradigma hidráulico, incrementando el suministro de agua y entendiendo la gestión del agua desde una lógica productivista, de acuerdo a los intereses de las élites socio-económicas. La construcción de desaladoras ha creado un marco hídrico descentralizado con una creciente participación del sector privado, pero manteniendo las políticas de aumento de la oferta de recursos hídricos lideradas por el Estado 
(Swyngedow, 2013, 2014; March, 2015). Otras investigaciones inciden en otros aspectos, como la influencia política que los territorios ubicados en torno a la cuenca del Ebro ejercieron sobre el nuevo gobierno elegido en 2004 para abolir el trasvase del Ebro e implantar el Programa AGUA de manera unilateral sin la participación y rigurosidad técnica que exige una intervención de esa magnitud (Gil y Rico, 2007; Rico, 2010).

Un segundo recurso no convencional gestionado de manera centralizada es la reutilización de las aguas residuales regeneradas. En la Comunidad Valenciana y Murcia, se ha producido un notable desarrollo de las infraestructuras de depuración (Pérez et al., 2014; Rico y Hernández, 2008); siendo éstas redimensionadas en los últimos años para aumentar los caudales regenerados de acuerdo al aumento poblacional experimentado en las últimas décadas. Esto ha permitido que la Demarcación Hidrográfica del Segura se sitúe como la única demarcación hidrográfica española donde se depura y reutiliza cerca del 100\% de las aguas residuales (Olcina y Moltó, 2010). Este sistema, empleado en este territorio desde hace años (Rico et al., 1998), permite optimizar el recurso obteniéndose un agua que según el grado del tratamiento se puede usar en función de su calidad para usos agrícolas (el mayoritario), pero también para el riego de campos de golf y jardines o el baldeo de calles. Su incorporación al ciclo hídrico ha permitido reservar el agua de mejor calidad, como las procedentes de fuentes convencionales, para usos urbanos y turísticos (Rico et al., 2013); disminuyéndose así la dependencia de las aguas del trasvase Tajo-Segura. Por todo ello, la reutilización de aguas regeneradas es considerada una de las prácticas que mejor se adapta a los principios del desarrollo sostenible (Seguí, 2004), aunque su uso esté limitado por la normativa y por el rechazo social que produce su utilización para usos domésticos por motivos sanitarios (Baeza, 2009; March et al., 2015a).

Paralelamente, otras alternativas descentralizadas a escala local como el aprovechamiento de aguas pluviales o la reutilización de aguas residuales a nivel de hogar están emergiendo, pero la introducción de infraestructuras a micro-escala exige una mayor participación de los ciudadanos y un fuerte apoyo gubernamental para un crear un nuevo modelo de gobernanza que transforme el actual régimen de gestión hídrica (Domènech, 2011; Domènech et al., 2013).

Esta investigación analiza la evolución del ciclo hidrosocial de Torrevieja, a partir del marco teórico que proporciona la Ecología Política Urbana, y concretamente durante los años 20002014 en los que el crecimiento urbanístico y demográfico ha generado significativos cambios en los flujos hídricos. Para ello, se describirá el ciclo hidrosocial de esta ciudad, analizando el recorrido y evolución de los flujos hídricos a través del sistema urbano, que es entendido como una construcción híbrida donde los factores naturales y los sociales se interrelacionan. Consecuentemente, será necesario analizar los principales procesos por los cuales el agua se metaboliza en el entorno urbano, las entradas, los consumos y las salidas; así como cuantificar los flujos hídricos en cada fase. Asimismo, se examinará los actores implicados en este ciclo, así como la influencia que ejercen sobre el conjunto del ciclo hidrosocial. Torrevieja supone un buen ejemplo de las intensas y rápidas transformaciones territoriales producidas durante el boom inmobiliario en uno de los territorios más áridos de la costa mediterránea española y cómo el incremento de las demandas ha motivado políticas de incremento de la oferta de agua basadas fundamentalmente en el desarrollo de las fuentes de suministro de agua no convencionales.

El artículo está organizado de la manera siguiente. Después de esta introducción, se analiza el contexto territorial de Torrevieja, explicando la evolución urbanística y poblacional de la ciudad 
durante las últimas décadas. Posteriormente, se caracterizan los componentes de su ciclo hidrosocial y se analizan los cambios producidos en él en el siglo XXI, examinando especialmente la introducción de los recursos hídricos procedentes de la desalación y la reutilización, así como los diferentes actores involucrados en la gobernanza del agua a nivel regional y local. Por último, se plantean las conclusiones del trabajo y los interrogantes que surgen ante la evolución futura de este ciclo hidrosocial.

\section{Boom inmobiliario y turismo residencial: dos décadas de transformaciones territoriales}

La provincia de Alicante durante el llamado boom inmobiliario, que abarcó el periodo 19972006, se convirtió en la cuarta provincia española donde más viviendas fueron construidas (Burriel, 2008; Hernández et al., 2015). El litoral mediterráneo concentró buena parte de ese desarrollo inmobiliario siendo Torrevieja un buen ejemplo de ello, ya que entre 1993 y 2002 se situó como el segundo municipio español tras Madrid en el número total de certificaciones de fin de obra, con 43.268 unidades construidas (Rico y Hernández, 2008). Su estructura urbana ha ido evolucionando hacia un modelo de tipo turístico-residencial con la construcción de numerosas urbanizaciones de tipo compacto y de baja densidad (Figura $N^{\circ} 1$ ). Este modelo urbano consume gran cantidad de suelo y repercute en un mayor consumo hídrico por la mayor presencia de usos exteriores (Hof y Schmitt, 2011; Mazón y Aledo, 2005; Rico et al., 2009).

Figura No 1

Evolución de la cobertura del suelo residencial 1990-2006

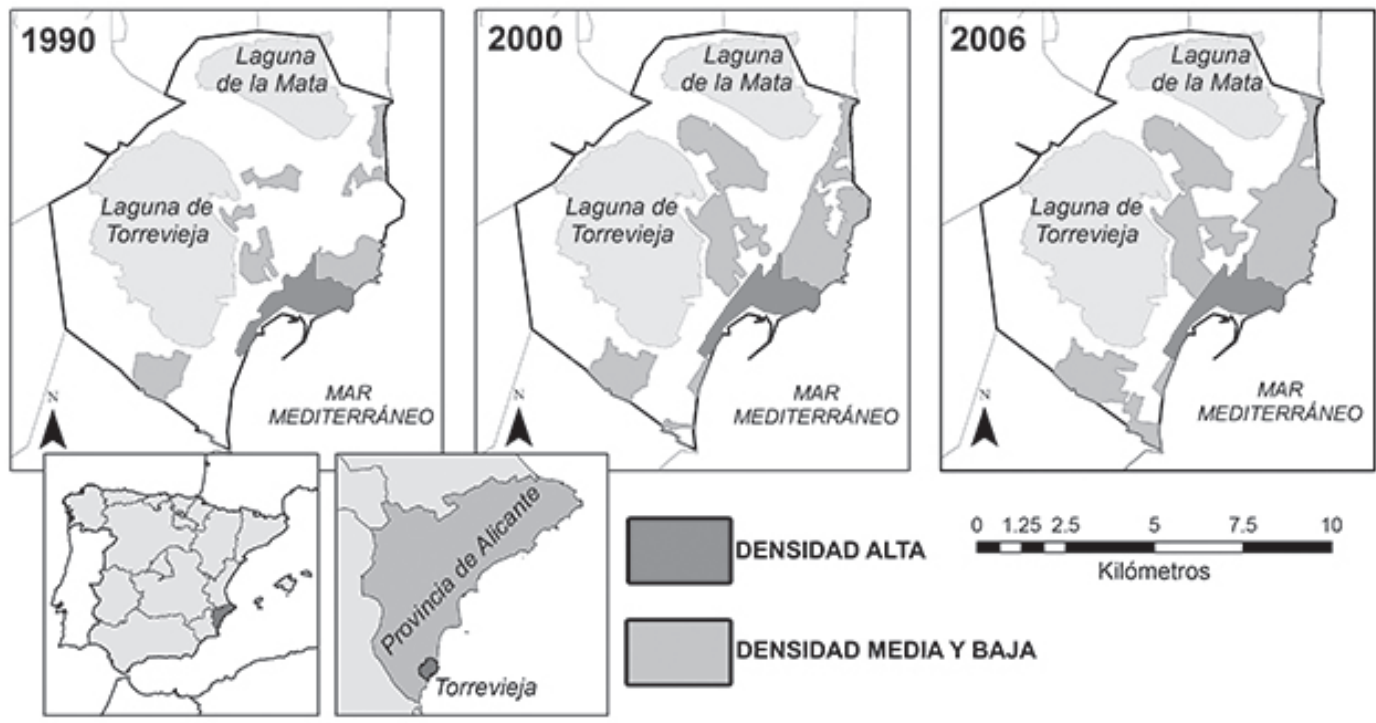

Fuente: Elaboración propia. 
El predominio de la tipología edificatoria de urbanizaciones de adosados es el resultado de una estrategia implementada desde la década de los setenta por los promotores inmobiliarios para la construcción de un mayor número de viviendas, a precio más reducido que los chalés, para compradores extranjeros de renta media-baja (Vera, 1987). Este hecho se plasma en la superficie ocupada por urbanizaciones de adosados, que representa el $36,30 \%$ del suelo urbanizado (Morote, 2014). Esta estrategia comercial también se plasmó en las urbanizaciones de baja densidad, ya que los chalés en el litoral sur de Alicante presentan unas parcelas de dimensiones reducidas $\left(250 \mathrm{~m}^{2}\right)$, si los comparamos con la superficie media de las parcelas del litoral norte provincial $\left(1.000 \mathrm{~m}^{2}\right)$ (Morote, 2014).

El crecimiento urbanístico continuó tras el primer boom de los setenta. Comparando los datos obtenidos de los últimos cuatro censos nacionales de población y vivienda (Cuadro No 1), se puede observar cómo el número de viviendas ha pasado de 19.517 en 1981 a 122.327 en 2011, corroborando el alto porcentaje de viviendas secundarias y vacías, base principal de este modelo turístico-residencial, donde la planta hotelera tiene un carácter testimonial. Este elevado número de segundas viviendas permite que se superen los 400.000 habitantes en el mes de agosto, reduciéndose esta cifra de forma drástica en temporada baja (Vera, 2002), donde la población censada se sitúa en unos 90.000 habitantes (INE, 2014).

\section{Cuadro No1}

Evolución del parque de viviendas, por tipologías, para la ciudad de Torrevieja

\begin{tabular}{|c|l|r|r|}
\hline & & No & porcentaje (\%) \\
\hline \multirow{3}{*}{1981} & Viviendas Principales & 3.529 & 18 \\
& Viviendas Secundarias/Vacías & 15.988 & 82 \\
& Total & 19.517 & 17 \\
\hline \multirow{3}{*}{1991} & Viviendas Principales & 8.831 & 83 \\
& Viviendas Secundarias/Vacías & 44.378 & 22 \\
& Total & 53.209 & 78 \\
\hline \multirow{3}{*}{2001} & Viviendas Principales & 21.565 & 33 \\
& Viviendas Secundarias/Vacías & 80.790 & 67 \\
& Total & 102.355 & \\
\hline \multirow{3}{*}{2011} & Viviendas Principales & 39.55 & \\
& Viviendas Secundarias/Vacías & 82.472 & \\
& Total & 122.327 & \\
\hline
\end{tabular}

Fuente: Elaboración propia basándose en INE, 2014.

El análisis de la evolución de la población pone de manifiesto que ésta comenzó a aumentar rápidamente durante la década de 1990, lo que se vincula al predominio de un turismo residencial en el que prevalecían las segundas residencias adquiridas por turistas nacionales. Esta situación comienza a cambiar a finales de los años noventa, cuando la población extranjera, constituida principalmente por británicos, alemanes, suecos y rusos, comienza a adquirir viviendas que ocupan de manera permanente. Esta dinámica se refleja en el porcentaje que este colectivo representa sobre el total, que representa el 18,4\% de la población censada en 1991, el 34,54\% en 2001 y el 53,5\% en 2013 (INE, 2014). En definitiva, las intensas transformaciones territoriales asociadas al 
crecimiento urbanístico provocaron que entre 1991 y 2008, año de inicio de la crisis económica, la población en Torrevieja se multiplicase por cuatro.

La extensión del hábitat turístico-residencial, una elevada densidad y ocupación estacional y el alto porcentaje de población extranjera plantearon serios problemas en el sistema de abastecimiento de agua potable desde el comienzo de la actividad turística en el municipio. La búsqueda de recursos para satisfacer unas demandas cada vez mayores ha sido una constante a lo largo del tiempo. En 1965, debido al inicio de la actividad turística, se agudizan los problemas de abastecimiento a la población que, hasta esos momentos, era de base agrícola y pesquera. Para intentar solucionarlos, se creó la "Comisión Administrativa para el Abastecimiento de Aguas a Torrevieja", encargada de realizar las obras de captación y ampliación de redes y depósitos necesarios para asegurar el suministro. No obstante, los problemas de escasez de recursos y de inseguridad del abastecimiento, especialmente en los meses de verano no se solventaron hasta la incorporación de la ciudad a la Mancomunidad de los Canales del Taibilla (MCT) en 1972 (MCT, 2009). Además, el modelo turístico adoptado ha conducido a unas intensas fluctuaciones demográficas estacionales, que afectan directamente a la demanda de agua, registrándose consumos hasta tres veces mayores durante la estación estival. Esta estacionalidad ha sido determinante a la hora de dimensionar las redes de abastecimiento y saneamiento con el fin de asegurar el suministro y tratar el agua residual generada. A pesar de estas características los módulos de consumo son bastante menores a los de otros núcleos turístico-residenciales de la costa alicantina, situándose en torno a los 200 litros/habitante/día para el mes de agosto con ocupaciones que no suelen superar los 90 días (Gil y Rico, 2007). Esto se relaciona con el predominio de bloques de apartamentos y viviendas unifamiliares en adosados, que presentan unos menores consumos para usos exteriores. Las dimensiones reducidas de las parcelas no suelen posibilitar la instalación de jardín y/o piscinas y, en el caso de que aparezcan, suelen ser de uso comunitario (Morote y Hernández, 2014).

\section{El ciclo hidrosocial de Torrevieja: cambios y nuevas tendencias}

Para describir el ciclo hidrosocial de Torrevieja es necesario entender los procesos metabólicos que lo dominan, la cuantía y la dirección de los flujos hídricos, los actores implicados en la gobernanza del agua y la influencia que ejercen sobre el conjunto del ciclo hidrosocial. En primer lugar, los aportes de agua que entran en el ciclo tienen diferentes procedencias y tipologías. Por un lado, los naturales que provienen de las aguas del trasvase Tajo-Segura, del río Taibilla y, en menor medida, del aporte procedente del agua de lluvia, de poca magnitud en esta zona. Éstos se completan, con la incorporación del agua embotellada como aporte externo. Estas aguas, una vez consumidas por los diferentes sectores de la población, son depuradas y vuelven a incorporarse al ciclo hidrosocial siendo utilizadas, de nuevo, para satisfacer otros usos como el agrícola.

En el año 2000, el modelo urbano turístico-residencial ya estaba consolidado, la ciudad contaba con casi 50.000 habitantes censados y continuaba su expansión. El ciclo hidrosocial a comienzos de esta década se sintetiza en un diagrama (Figura No 2), donde se desglosan las entradas, los consumos y salidas de flujos hídricos para usos urbanos, así como los diferentes procesos por los que pasa el agua y las empresas gestoras en cada fase, tratando con ello poner de manifiesto el "complejo metabolismo entre la naturaleza y la sociedad" (Johnston et al., 2000: 590). 
Figura $\mathrm{N}^{\circ} 2$

Diagrama de flujos de la ciudad de Torrevieja en 2000

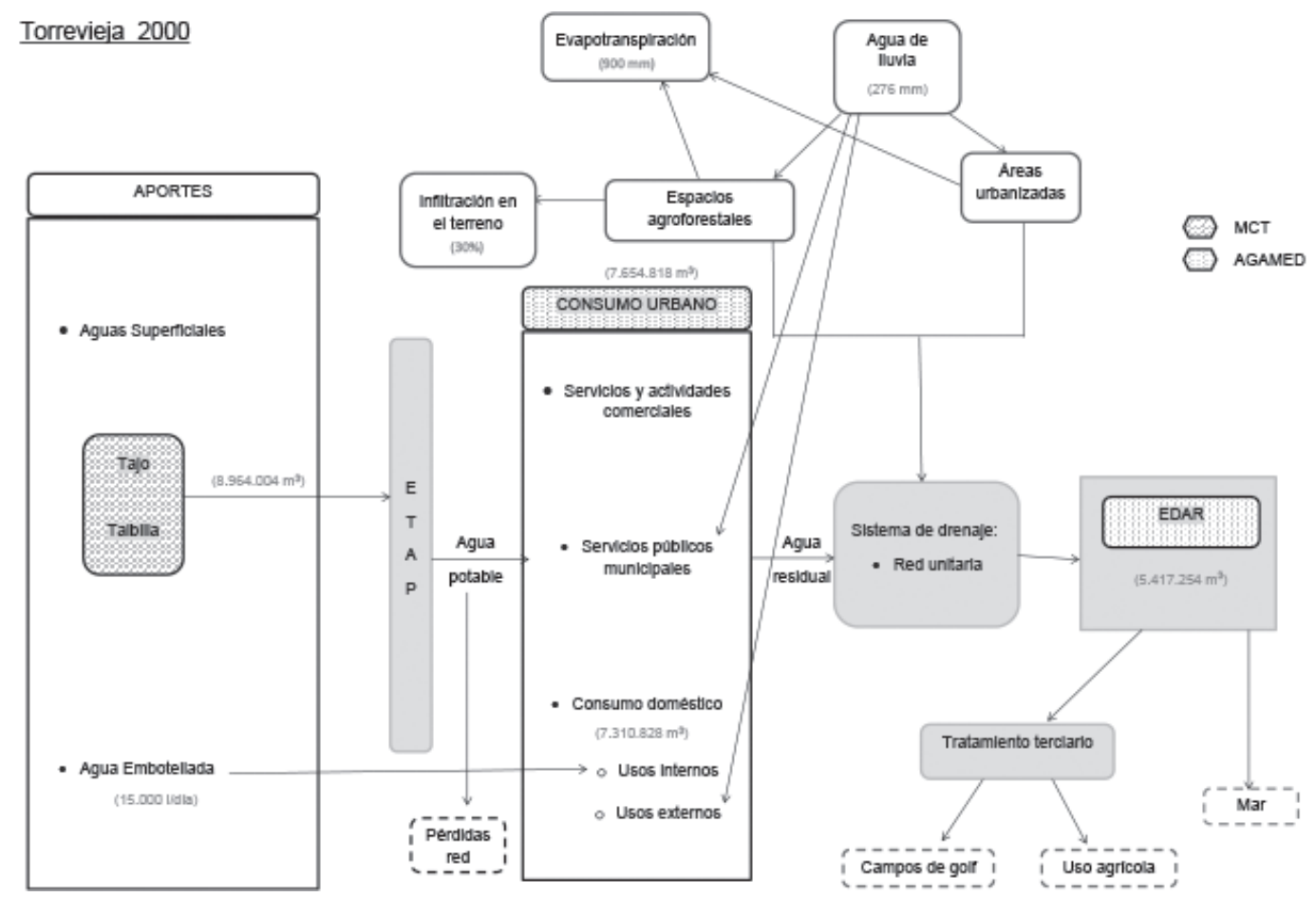

Fuente: Elaboración propia basándose en MCT, EPSAR, AGAMED, Ayuntamiento de Torrevieja.

Teniendo en cuenta la estructura descrita en el párrafo anterior, desde el punto de vista de las entradas, el principal aporte al sistema procede de la MCT, organismo público que se encarga del suministro del agua en alta a escala regional. Este organismo abastece a más de 2.500 .000 habitantes, además de otro millón durante el verano por efecto del poblamiento estacional vinculado al turismo, principalmente en las provincias de Murcia y el sur de Alicante (Gil y Rico, 2007). Esta entidad recibe agua de diversas fuentes; siendo las principales el río Taibilla y el trasvase Tajo-Segura. El porcentaje de agua suministrado por cada una varía anualmente dependiendo de coyunturas ambientales y de las demandas del momento. El trasvase Tajo-Segura, que funciona desde 1979, suministra un promedio de $321 \mathrm{hm}^{3} /$ año de los cuales la tercera parte se destina a demandas urbanas, lo que representa el $60 \%$ de los recursos que gestiona la MCT. El agua procedente de la MCT llega al sistema urbano de Torrevieja, previo paso por la estación de tratamiento de agua potable (ETAP) preparándola para el consumo. El volumen suministrado en el año 2000 a Torrevieja por parte de la MCT fue de $8.964 .004 \mathrm{~m}^{3}$. En la escala municipal, o en baja, la gestión del ciclo integral del agua es llevada a cabo por la empresa mixta Aguas del Arco Mediterráneo (AGAMED), fundada en 1999 y formada en un $26 \%$ por el ayuntamiento de Torrevieja y en un $74 \%$ por Hidraqua, filial del Grupo Aquadom (propiedad de la multinacional francesa Suez) para la gestión del agua en la Comunidad Valenciana.

Junto a estos recursos convencionales hay que tener en cuenta otro aporte hídrico, el agua embotellada consumida por la población. Dado que el tipo de agua de la zona es calificada como 
"dura" por su alto contenido en carbonato cálcico, es muy común en los hogares del litoral mediterráneo el consumo de agua embotellada, pero el cálculo de este consumo es bastante complejo. En este estudio, se ha realizado una estimación teniendo en cuenta el número de litros de agua embotellada consumida al año por habitante, que asciende a 101 litros conforme a los datos proporcionados por la Asociación Nacional de Empresas de Aguas de Bebida Envasada (2013).

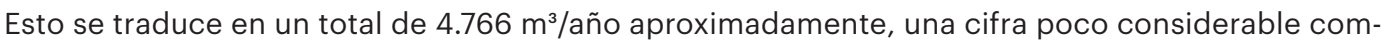
parada con lo aportado por otras fuentes. Otro aporte que tomar en consideración, aunque en esta zona sea muy escaso, es el agua de lluvia que entra al sistema. Dado que el modelo urbano es de baja densidad se considera que, de los $276 \mathrm{~mm}$ de agua que cayeron en 2000, aproximadamente un $40 \%$ se infiltró, incorporándose el 60\% restante al sistema (Aparicio, 1999: 210), ya sea como regadío de las zonas verdes o como agua de escorrentía que desemboca en la red de saneamiento.

El segundo elemento analizado del ciclo hidrosocial es el consumo urbano del agua, que abarca distintos usos, como el uso municipal, el comercial y el uso doméstico de agua. En el año 2000, el consumo doméstico ascendía a $7.310 .828 \mathrm{~m}^{3}$, lo que supone que el módulo de consumo se situaba en 399 litros/habitante/día, una cifra considerablemente alta. Este análisis en parte se ve dificultado por la falta de datos reales sobre la evolución de la población flotante que reside temporalmente en Torrevieja, lo que completaría el análisis basado simplemente en la evolución de la población empadronada. Además, la existencia de una tarifa única para los distintos usos urbanos impide diferenciar entre usos domésticos y comerciales, que conjuntamente suponen entre el 93 y el $96 \%$ del consumo urbano del agua en Torrevieja para el periodo analizado (2000-2014), lo que corrobora la trascendencia del modelo turístico-residencial.

El tercer elemento a considerar en este ciclo hidrosocial son las salidas del sistema. La red de drenaje es unitaria, es decir, tiene un solo conducto para transportar las aguas residuales y las aguas pluviales, por lo que toda esta agua llega a la Estación Depuradora de Aguas Residuales (EDAR) de Torrevieja. Esta planta, tras su ampliación entre 2005 y 2007 por la Entidad Pública de Saneamiento de Aguas Residuales de la Comunidad Valenciana (EPSAR), ha pasado de tener una capacidad de $21.000 \mathrm{~m}^{3} /$ día a tratar hasta $60.000 \mathrm{~m}^{3} /$ día de aguas residuales, adecuándose así a los picos de caudal asociados a la llegada de los turistas y dando servicio a un total de 490.000 habitantes equivalentes. La normativa que regula el tratamiento, gestión y posterior reutilización de las aguas residuales juega un papel clave en la evolución de estos procesos. A nivel europeo, la implantación de la Directiva Marco del Agua 2000/60/CEE y la Directiva 271/91/CEE para el tratamiento de las aguas residuales han marcado un antes y un después en los procesos de depuración. Siendo estos postulados recogidos a nivel regional por el Plan Director de Saneamiento y Depuración de la Comunidad Valenciana de 1992 y la Ley de Saneamiento de las aguas Residuales de 1992. Además, ante la ausencia de normativa europea para la reutilización de las aguas residuales, en España se aprobó el Real Decreto 1620/2007, que regula la reutilización de estas aguas a nivel nacional. Ante este marco legal se tienen que someter todos los actores involucrados en el ciclo hidrosocial del agua (políticos, usuarios, gestores públicos y privados) independientemente de su procedencia e intereses.

La totalidad del agua depurada es regenerada mediante un tratamiento avanzado consistente en una filtración rápida en lechos pulsantes de arena y desinfectada mediante radiación ultravioleta. El uso de estas aguas regeneradas, que durante la pasada década ya se empleaba en un alto porcentaje, alcanza en 2013 el 100\% del volumen tratado. El 94\% se destina para riego agrícola, 
mediante concesiones administrativas otorgadas por la Confederación Hidrográfica del Segura a la Comunidad de Regantes de Torremiguel. La escasez de recursos ha determinado que ya desde finales de los años ochenta los agricultores hayan recurrido a este tipo de agua (Rico et al, 1998). El $6 \%$ del agua restante recibe un tratamiento terciario por infiltración-percolación modificada y desinfección por cloración y, posteriormente, se reutiliza para uso municipal en riego de parques y jardines, mediante una compleja red de distribución. Las aguas depuradas procedentes de la estación de tratamiento de aguas residuales de Torrevieja son almacenadas temporalmente en un depósito de regulación propiedad de la Comunidad de Regantes de Torremiguel e impulsadas a depósitos intermedios que dicha comunidad tiene distribuidos por el Alto de las Majadas, en el término municipal vecino de Orihuela.

\section{Tendencias de cambio}

Las tendencias de cambio se evidencian en una serie de cuestiones como son la evolución del consumo y la introducción de fuentes de agua no convencionales en el ciclo hidrosocial. En este apartado se analizará el efecto de esas dinámicas en los diagramas de flujos.

El análisis de la evolución de los consumos urbanos permite diferenciar tres etapas de acuerdo con las tendencias observadas (Figura $N^{\circ} 3$ ). La primera corresponde a la etapa comprendida entre 2000 y 2003. Ésta enlaza con un crecimiento continuado de las demandas hídricas en paralelo al crecimiento residencial y demográfico que registra este municipio desde 1981. Torrevieja entre 2000 y 2003 incrementa su población en unos 27.000 habitantes al pasar de 50.189 a 77.943 personas. Esta dinámica se refleja en los volúmenes suministrados por la MCT, aunque con menor intensidad, ya que, mientras que la población crece un 55\% el agua suministrada lo hace un $16 \%$.

Figura $\mathrm{N}^{\circ} 3$

Evolución del volumen suministrado y facturado en Torrevieja

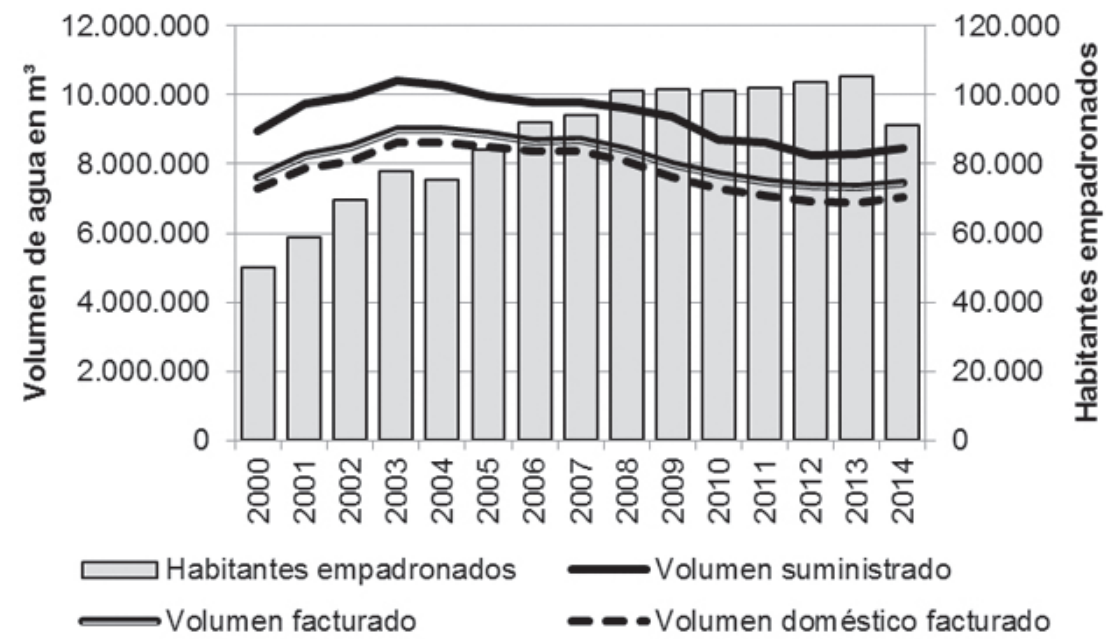

Fuente: Elaboración propia basándose en INE y AGAMED. 
El año con el mayor aporte suministrado de todo el periodo analizado, y de toda la historia de Torrevieja, fue 2003 con 10.427,710 m³. Un año después y en base a estas tendencias de crecimiento que se registraba en la mayoría de las ciudades de la costa mediterránea (March et al., 2014) se redactaba el Programa AGUA.

Sin embargo, a partir de 2003 se inicia una inflexión en la curva de la demanda que marca el inicio de una segunda etapa caracterizada por una ligera disminución del consumo, que se prolonga entre 2004 y 2007. Esta tendencia negativa, no obstante, no va asociada a una pérdida de población, sino todo lo contrario, ya que se mantiene el crecimiento demográfico iniciado en la etapa anterior. Una de las razones de este descenso va vinculada a las mejoras técnicas y control de fugas efectuado en la red de distribución por parte de AGAMED que redunda en una mejora del rendimiento hidráulico. Éste, que a principios de la década se situaba en torno al $85 \%$, se eleva al $89 \%$ en 2004. Otro factor que influye en el descenso del consumo en esta etapa es el creciente uso de las aguas residuales regeneradas para usos municipales, agrarios y riego de campos de golf, jardines y parques, que pasan de representar el 1,8\% del agua facturada en el 2000 al $4,5 \%$ en el 2013 según datos de EPSAR, lo que se traduce en una detracción de volúmenes de mayor calidad suministrados por la MCT. A estos factores, se une la generalización de dispositivos de ahorro tanto en los sistemas de grifería como en los electrodomésticos (Gil et al., 2015) y la realización de campañas de concienciación ambiental (March et al., 2015b). Este descenso del consumo se traduce en la evolución de la dotación por habitante y día, que ha pasado de 399 litros en el año 2000 a 243 en el año 2007.

La tercera etapa se inicia en 2008 y se prolonga hasta la actualidad y está caracterizada por un descenso del consumo más intenso; tendencia que se repite en otras ciudades españolas (Gil et al., 2015) y, en general, en ciudades de los países occidentales (March y Saurí, 2014). El estallido de la burbuja inmobiliaria marca el inicio de la crisis económica a finales de 2007, provocando el incremento del paro y la reducción de la capacidad adquisitiva de las familias, el estancamiento demográfico y el aumento del precio del agua durante los últimos años. Éstos factores, junto con los ya comentados de la etapa anterior, explican que entre el año 2000 y el 2013 el volumen suministrado haya disminuido un $7,59 \%$ y el consumo urbano de agua un $4,24 \%$.

Una década después, y a pesar del sostenido incremento demográfico y urbanístico, el diagrama de flujos no ha variado sustancialmente (Figura $N^{\circ} 4$ ). Los principales cambios se asocian a la incorporación del agua desalinizada como fuente de suministro en la MCT. Concretamente, desde el 2005, se incluyen las aguas desalinizadas procedentes de la desaladoras de San Pedro del Pinatar I y II (Rico y Hernández, 2008), proporcionando una capacidad máxima de $48 \mathrm{hm}^{3}$ / año (March et al., 2014; Rico, 2014). Esta estrategia de aumento y diversificación de las fuentes de suministro se completaría con la introducción a la MCT de los caudales de las plantas proyectadas por el Programa AGUA como es el caso de la planta de Torrevieja.

La incorporación del agua desalada al sistema fue debida a la reducción de los recursos superficiales en la cabecera del Tajo, que se tradujeron en una disminución de los aportes del trasvase Tajo-Segura, como consecuencia de la sequía de 2005-2009. En 2012, para toda el área abastecida por la MCT, se inyectaron en la red $44 \mathrm{hm}^{3}$ de agua desalada procedente de las plantas de Alicante I y San Pedro del Pinatar I (Figura No 5). Tras la recuperación de un ciclo hidrológico normal, su consumo se reduce significativamente. Así en 2013, para el conjunto de la MCT supusieron tan solo el $3,26 \%$ del total de agua suministrada (MCT, 2013). 
Figura $\mathrm{N}^{\circ} 4$

Diagrama de flujos de la ciudad de Torrevieja en 2013

Torrevieja 2013

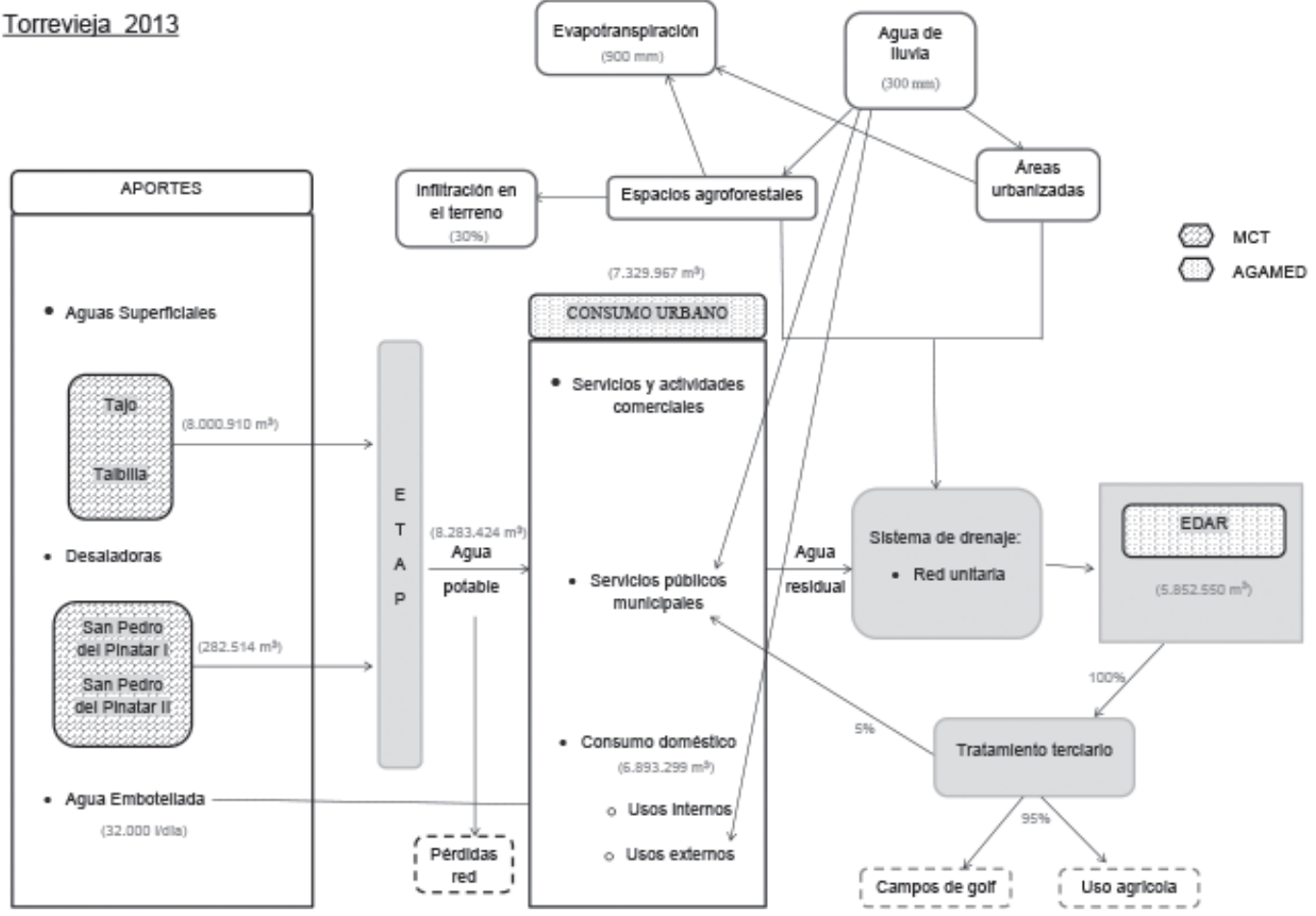

Fuente: Elaboración propia basándose en MCT, EPSAR, AGAMED, Ayuntamiento de Torrevieja.

Figura No 5

Procedencia de las aguas suministradas por la MCT (1989-2013)

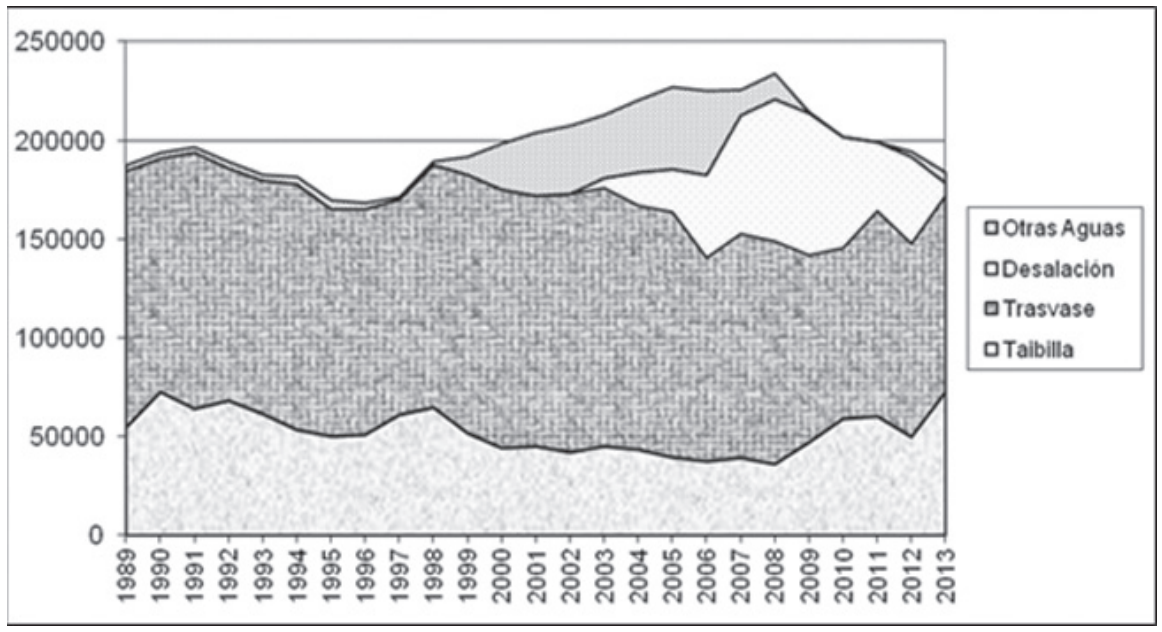

Fuente: Rico, 2014. 
La desaladora de Torrevieja, con una producción teórica de 80 hm³/año, es la planta de mayor capacidad proyectada por el Programa AGUA, así como la mayor de toda Europa y la segunda del mundo con tecnología de osmosis inversa. Ha supuesto una inversión que ronda los 300 millones de euros, a los que hay que sumar unos costes de conservación y mantenimiento que superan los 56 millones de euros al año, representando la mitad de ese total los costes energéticos (Del Villar, 2014). Su explotación y mantenimiento ha sido gestionado por ACUAMED, organismo público responsable de implementar el Programa AGUA, usando la fórmula de concesión administrativa durante 15 años a una Unión Temporal de Empresas (UTE)5. A pesar de que su construcción comenzó en 2006, su puesta en marcha se ha retrasado por diversos factores, entre los que cabe citar las disputas políticas entre el gobierno central y el autonómico, con diferentes intereses en relación al uso de las aguas desaladas, las dificultades para asegurar el suministro eléctrico necesario y la caída de la demanda; no entrando en funcionamiento hasta agosto de 2015, coincidiendo con un periodo de sequía. Actualmente el agua que genera, unos $24 \mathrm{hm}^{3} / a n ̃ o$, no está siendo inyectada a la red de la MCT, puesto que ésta ya tiene cubierta su capacidad de $170 \mathrm{hm}^{3}$ para usos urbanos, sino que se está derivando hacia el embalse de La Pedrera, siendo su destino el riego agrícola. El retraso en su puesta en funcionamiento y el reducido porcentaje de agua generada pone de manifiesto la infrautilización de estas plantas como consecuencia de la caída de la demanda, es decir, se ha pasado de una situación de déficit hídrico a otra excedentaria y ello a pesar de que el potencial máximo de desalación está lejos de verse implementado (March et al., 2014).

La infrautilización de este recurso no solo se explica por la disminución de la demanda, sino también por su elevado precio, lo que determina que sea el recurso al que se recurre en último lugar. Sin embargo, ello no excluye que sea un recurso estratégico en zonas de déficit hídrico. El alto coste energético que conlleva el proceso de desalinización, que supone en torno al $48 \%$ de los costes totales de producción (Del Villar, 2014:108), explica la diferencia en su precio frente a otras fuentes de suministro. La reforma del mercado eléctrico en España, llevada a cabo en 2008 a instancias de la Unión Europea (March et al., 2014), supuso un aumento del precio de la electricidad en torno al 64\% entre 2007 y 2012 (Del Villar, 2014:108). Esto ha provocado que el coste total medio para el agua desalada producida en las desaladoras de la MCT en 2012 ascendiera a 0,620 $€ / \mathrm{m}^{3}$. El precio de la energía unido a la situación de infrautilización de la capacidad máxima de producción redunda en un mayor precio del metro cúbico producido, ya que "la repercusión de los costes fijos es proporcional al empleo de la capacidad de producción de la planta" (Del Villar, 2014: 110). Si tenemos en cuenta que, a principios del año 2014, la capacidad de producción de la planta de Torrevieja se situaba en torno a un 20-30\%, el coste total de producción de un metro

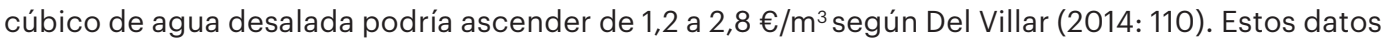
contrastan con el precio final manifestado por Acciona, la empresa gestora de la desaladora de Torrevieja, que en junio de 2016 se situaba alrededor de $0,5 € / \mathrm{m}^{3}$. Aun así, estos precios comparados con los del agua del trasvase Tajo-Segura, de $0,1157 € / \mathrm{m}^{3}$, resultan prohibitivos para los regantes (Rico, 2010), y también han tenido significativas repercusiones en los precios del agua para los usos urbanos. En el caso de Torrevieja, desde la introducción del agua desalada en la MCT la tarifa del agua para los abonados urbanos ha aumentado un $63,3 \%$ en todos los bloques de consumo entre 2004 y 2015 (Boletín Oficial de la Provincia de Alicante, 2004 y 2015). 


\section{Influencias de la gobernanza sobre el ciclo hidrosocial}

La escasez de recursos hídricos se configura como un marco favorable a la acumulación de capital y la expansión de los mercados (March y Saurí, 2014), como corrobora el ejemplo de Torrevieja. Desde la incorporación de Torrevieja a la MCT, se produjo la participación del capital privado en la gestión hídrica. Entre 1973 y 1999, el servicio de abastecimiento municipal era gestionado únicamente por Aquagest Levante, empresa privada que en 2013 cambia de denominación a la actual Hidraqua. La necesidad de contar con financiación a escala municipal para hacer frente a la ampliación o mantenimiento de la red de distribución durante el proceso de urbanización y el aumento de los ingresos en los ayuntamientos mediante la privatización de determinados servicios explica el incremento de empresas privadas en la gestión del suministro de los ciclos hidrosociales en gran parte del litoral mediterráneo español desde finales de los años noventa (Del Romero, 2006). Según el estudio de Del Romero (2006), la tendencia al alza en la gestión privada del agua a nivel municipal se localiza, sobre todo, en aquellos entornos de reciente y rápida urbanización, como sucede con el litoral mediterráneo.

Desde el año 1999, es la empresa mixta AGAMED la que gestiona la distribución, el alcantarillado y la depuración en Torrevieja. Ha llevado a cabo considerables inversiones en la red de distribución, ya que parte de sus beneficios dependen de minimizar las pérdidas de la red; así como la incorporación de "nuevos caudales" al sistema de flujos (aguas regeneradas). Sin embargo, ha sido el Estado el que ha asumido los riesgos financieros más elevados por la construcción de las grandes infraestructuras hídricas mientras que, en comparación, las inversiones de ampliación o mantenimiento de la red a nivel municipal representan un coste muy inferior (March y Saurí, 2014). Concretamente en el caso de Torrevieja, las grandes infraestructuras financiadas por el Estado para asegurar la llegada de flujos hídricos, como el trasvase Tajo-Segura o las sucesivas ampliaciones que se han producido en la EDAR, han sido ejecutadas con fondos públicos.

La falta de planificación en cuanto a la necesidad de la construcción o el dimensionamiento de la desaladora de Torrevieja ha generado, a su vez, dos consecuencias de signo inverso para la esfera pública y la privada. Para la primera, los resultados de esta actuación han sido la generación de una deuda elevada con entidades de crédito de organismos públicos como ACUAMED fruto del mantenimiento e infrautilización de las desaladoras. Este organismo en 2011 reconocía una deuda de 326 millones de euros que había crecido frente a los 23 de 2009 (Rico, 2014). A esta situación hay que añadirle los últimos casos de corrupción investigados en torno a esta empresa pública por presuntos fraudes en la concesión de los contratos y en los sobrecostes de algunas obras encargadas a algunas de las empresas líderes en el sector de la construcción, entre los que se encuentra la desaladora de Torrevieja. Esta coyuntura se ha extendido a otros organismos públicos, como la MCT, que ha visto cómo se multiplicaba por cinco su deuda entre 2004 y 2012, debido a la suscripción de convenios con ACUAMED para adquirir la producción de las desaladoras de Valdelentisco, Torrevieja y Águilas, ya que, aunque la producción de agua desalada sea mínima, la MCT ha de hacer frente a los gastos fijos de operación y mantenimiento, más los generados por la amortización de las obras (Rico, 2014). Esta situación incluso podría agravarse por la posibilidad que la Unión Europea obligue a devolver los fondos europeos utilizados para implementar el Programa AGUA por la infrautilización de las desaladoras. Esto reducirá su capacidad de maniobra en los próximos años y trasladaría a los precios del agua los costes de la financiación de estas obras a los abonados urbanos (Del Villar, 2014); lo que en parte ya se está produciendo. 
Sin embargo, para la esfera privada, el resultado del Programa AGUA en el litoral mediterráneo ha beneficiado a las grandes empresas constructoras y sus filiales en el sector del agua ${ }^{6}$ con la construcción de desaladoras. Los contratos multimillonarios de construcción y mantenimiento de estas infraestructuras suscritos con ACUAMED han posibilitado una expansión internacional posterior de estas grandes empresas que las han situado en una posición de liderazgo en el mercado global de la desalación (March et al., 2014; Swyngedouw, 2013).

\section{Conclusiones}

La complejidad de las interacciones entre flujos hídricos, gobernanza del agua y actores implicados en torno al abastecimiento de agua en territorios donde se ha producido una intensa transformación territorial como Torrevieja exige el estudio de sus relaciones a partir del concepto de ciclo hidrosocial. Los resultados de este análisis muestran cómo desde hace décadas, el Estado se ha encargado de asegurar el suministro de agua a través de la construcción de grandes infraestructuras hídricas y su redimensionamiento para adaptarse al aumento de la demanda y a la estacionalidad del consumo. La constitución de la Mancomunidad de los Canales del Taibilla y la construcción del trasvase Tajo-Segura sentaron las bases del desarrollo socio-económico de gran parte de las provincias de Murcia y sur de Alicante, donde se localiza la población de Torrevieja. En ambos casos, la finalidad de estas actuaciones era asegurar los recursos hídricos en contexto climático árido, que acentuaba la situación de déficit hídrico, mediante la construcción de infraestructuras que garantizasen las demandas. Este tipo de políticas, insertas en el denominado paradigma hidráulico, experimentan una creciente contestación al amparo de la Nueva Cultura del Agua, que aboga por paradigmas más sostenibles. La derogación del trasvase del Ebro y la promulgación del Programa AGUA ejemplifican este cambio, pero también la apuesta hacia un desarrollo sin precedentes de las fuentes de agua no convencionales. La reutilización de aguas residuales $y$, sobre todo la desalación, fueron planteadas como alternativas sostenibles a las tradicionales políticas hidráulicas para garantizar el suministro ante el continuo crecimiento urbanístico y demográfico.

En el caso de la reutilización, esta premisa se cumplió, ya que su incorporación ha aliviado la demanda sobre las aguas blancas, especialmente para usos agrícolas en la comarca de la Vega Baja del Segura. Una buena gestión del agua, donde el intercambio de usos permite sustituir aguas regeneradas por aguas de fuentes convencionales, libera volúmenes de agua de mayor calidad para usos prioritarios como el suministro de agua para el consumo humano, ya que la reutilización de aguas regeneradas de manera directa o indirecta aún no es viable para este uso por motivos legales, sanitarios y a la percepción negativa de los consumidores. En cambio, la introducción de los caudales desalados ha resultado más polémica. La deficiente planificación en cuanto a la dimensión de las plantas desaladoras no tuvo en cuenta un posible cambio en las tendencias de consumo urbano de agua y el elevado precio de la misma tanto para usos agrarios como urbanos, lo que ha derivado en su infrautilización. Esta situación augura un futuro escenario de disputas en torno al acceso a esta fuente de suministro por parte de los agricultores como consecuencia del aumento de las tarifas. La incertidumbre que supone el hecho de que los consumidores, sobre

Las principales filiales del agua y empresas matrices españolas en materia de desalación son: Aqualia-FCC; Pridesa-Acciona; Cobra-ACS; Sadyt-Sacyr; Inima-OHL; Befesa-Abengoa; Cadagua-Ferrovial Agroman. 
todo agrarios, puedan pagar el agua desalada pone en entredicho la rentabilidad de las infraestructuras construidas. Sin embargo, por otro lado, la posibilidad de que los caudales desalados vean aumentada su trascendencia a causa de la detracción del volumen del trasvase Tajo-Segura debido a futuras sequías o a conflictos con las regiones cedentes, así como la merma de los caudales del Taibilla, si se cumplen las previsiones sobre la reducción de los recursos hídricos como consecuencia del cambio climático (IPCC, 2014), las convierte en un recurso estratégico para este territorio, ya que le aseguraría el autoabastecimiento sin depender de aportes externos.

Este conflicto generado a partir de la puesta en marcha del Programa AGUA refleja, no obstante, la adaptación del tradicional paradigma hidráulico a un marco de gobernanza de agua más descentralizado. El aumento de la participación del sector privado en la gestión hídrica ha permitido que las élites socio-económicas, al mando de las grandes empresas de la construcción españolas, mantuvieran su crecimiento diversificando su actividad y expandiéndose internacionalmente gracias a la inversión pública, que, además, sostiene la deuda generada por estas infraestructuras sobredimensionadas. La introducción de los actores privados en la gestión de ciclos integrales del agua, que como la MCT antes eran enteramente públicos, plantea incógnitas en torno a la evolución de la gobernanza urbana del agua. Estas dudas se fundamentan en casos que actualmente se están dando en el norte del litoral mediterráneo español, donde la empresa pública catalana Aigües Ter-Llobregat, encargada del suministro de agua a nivel regional, se encuentra en proceso de privatización motivado por una elevada deuda con entidades financieras privadas (March y Saurí, 2013).

\section{Referencias bibliográficas}

AguAS DE LAS CUENCAS MEDITERRÁnEAS (ACUAMED). Memoria 2011 de la Sociedad Estatal Aguas de las Cuencas Mediterráneas. Madrid: Ministerio de Agricultura, Alimentación y Medio Ambiente, 2011.

APARICIO, F.J. Fundamentos de hidrología de superficie. México: Limusa, 1999.

ARAHUETES, A. Desalinización y energía en España. Situación y perspectivas. El caso del Mediterráneo. En: MORA, J. (editor). Gestión de recursos hídricos en España e Iberoamérica. Pamplona: Editorial ARANZADI, 2015, p.529-540.

ASOCIACIÓN NACIONAL DE EMPRESAS DE AGUAS DE BEBIDA ENVASADA, 2013. Disponible en Internet: http://www.aneabe.com/cifras_sector/produccionEspana

AYALA-CARCEDO, F. De la política hidráulica a la política del agua sostenible. Tecnoambiente, 1999, No 90, p. 5-9.

BAEZA, J. Reutilización de aguas residuales para riego. Alicante: Trabajo Fin de Máster en Gestión Sostenible y Tecnologías del Agua, Universidad de Alicante, 2009.

BOLETÍN OFICIAL DE LA PROVINCIA DE ALICANTE. Aquagest. Servicio municipal de agua potable. 2004, No 34. Disponible en Internet: http://www.dip-alicante.es/bop2/pdftotal/2004/02/11-02-04. pdf 
BOLETIN OFICIAL DE LA PROVINCIA DE ALICANTE. Información pública de tarifa de agua potable aplicable en el municipio de Torrevieja, 2015, № 108. Disponible en Internet: http://www.dip-alicante.es/bop2/pdftotal/2015/06/08_108/Sumario.pdf

BURRIEL, E. L. La "década prodigiosa" del urbanismo español (1997-2006). Scripta Nova. Revista Electrónica de Geografía y Ciencias Sociales, 2008, Vol. XII, № 270 (64). Disponible en Internet: http://www.ub.edu/geocrit/-xcol/383.htm

DEL ROMERO, L. Privatització de la gestió de l'aigua i govern del territori a l'Arc Mediterrani espanyol. Documents d'Anàlisi Geogràfica, 2006, No 48, p. 35-59.

DEL VILLAR, A. El coste energético de la desalinización en el Programa A.G.U.A.. Investigaciones Geográficas, 2014, № 62, p. 101-112.

DEMARCACIÓN HIDROGRÁFICA DEL SEGURA (DHS). Plan Hidrológico de cuenca de la Demarcación hidrográfica del Segura. Murcia: Ministerio de Agricultura, Alimentación y Medio Ambiente, 2014.

DOMENECH, L. Rethinking water management: From centralised to decentralised water supply and sanitation methods. Documents d'Anàlisi Geogràfica, 2011, Vol. 57, № 2, p. 293-310.

DOMĖNECH, L.; MARCH, H. y SAURÍ, D.: Degrowth initiatives in the urban water sector? A Social Multi-criteria Evaluation of non-conventional water alternatives in Metropolitan Barcelona. Journal of Cleaner Production, 2013, N 38, p. 44-55.

GENERALITAT VALENCIANA: II Plan Director de Saneamiento y Depuración de la Comunidad Valenciana, 2003. Disponible en Internet: http://www.agricultura.gva.es/agua/participacion-publica/ii-pii-plan-director-de-saneamiento-de-la-comunidad-valencianalan-director-de-saneamiento-de-la-comunidad-valenciana

GIL, A. Clima e hipótesis de cambio climático en la región geográfica del sureste ibérico. Investigaciones geográficas, 2009, № 49, p. 5-22.

GIL, A y RICO, A.M. El problema del agua en la Comunidad Valenciana. Valencia: Fundación de la Comunidad Valenciana Agua y Progreso, 2007.

GIL, A.; HERNÁNDEZ, M.; MOROTE, A.F.; RICO, A.M.; SAURÍ, D. y MARCH, H. Tendencias del consumo de agua potable en la Ciudad de Alicante y Área Metropolitana de Barcelona, 2007-2013. Alicante: Hidraqua, Gestión Integral de Aguas de Levante S.A. y la Universidad de Alicante, 2015.

GIORGI, F. \& LIONELLO, P. Climate change projections for the Mediterranean region. Global and Planetary Change, 2008, Vol. 63, N², p. 90-104.

HERNÁNDEZ, M.; SAURÍ, D. y MORALES, A. Auge y caída de nuevas naturalezas urbanas: plantas ornamentales y expansión turístico-residencial en Alicante. Boletín de la Asociación de Geógrafos Españoles, 2015, № 68, p. 129-157. 
HOF, A. \& SCHMITT, T. Urban and tourist land use patterns and water consumption: Evidence from Mallorca, Balearic Islands. Land Use Policy, 2011, Vol. 28, № 4, p. 792-804.

INSTITUTO NACIONAL DE ESTADÍSTICA (INE). Estadística del padrón continuo (2014) y Censos de población y vivienda. Varios años: 1981, 1991, 2001 y 2011. Disponible en Internet: http://www.ine. es/inebmenu/mnu_cifraspob.htm

INTERGOVERNMENTAL PANEL ON CLIMATE CHANGE (IPCC). Climate Change 2013 and Climate Change 2014. 2014. Disponible en Internet: http://www.ipcc.ch/

JOHNSTON, R.; GREGORY, D.; PRATT, G.; WATTS, M. \& SMITH, D. (editors). The Dictionary of Human Geography. Oxford: Blackwel, 2000.

LINTON, J. \& BUDDS, J. The hydrosocial cycle: Defining and mobilizing a relational-dialectical approach to water. Geoforum, 2014, N 57, p. 170-180.

MANCOMUNIDAD DE CANALES DEL TAIBILLA. El organismo y su evolución histórica. Albacete: Ministerio de Agricultura, Alimentación y Medio Ambiente, 2009. Disponible en Internet: https:// www.mct.es/files/Biblioteca/Organismo\%20y\%20su\%20evolucion/Organismo_y_su_evolucin_historica.pdf

MANCOMUNIDAD DE CANALES DEL TAIBILLA. Gestión del servicio 2013. Albacete: Ministerio de Agricultura, Alimentación y Medio Ambiente, 2013. Disponible en Internet: https://www.mct.es/ files/Biblioteca/memoria\%202013/Gestion\%20del\%20servicio\%202013.pdf

$\mathrm{MARCH}, \mathrm{H}$. Taming, controlling and metabolizing flows: Water and the urbanization process of Barcelona and Madrid (1850-2012). European Urban and Regional Studies, 2013, p. 1-18.

MARCH, H. The politics, geography, and economics of desalination: a critical review. WIREs Water, 2015, Vol. 2, No 3, p. 231-243.

MARCH, H. y SAURÍ, D. The unintended consequences of ecological modernization: debt-induced reconfiguration of the water cycle in Barcelona. Environment and Planning A, 2013, No 9, p. 20642083.

MARCH, H. \& SAURÍ, D. ¿Qué sostenibilidad hídrica? Una interpretación crítica del descenso reciente del consumo doméstico de agua en Barcelona. WATERLAT- GOBACIT Network Working Papers. Thematic Area Series SATCUASPE- TA3 - Urban Water Cycle and Essential Public Services, 2014, Vol. 1, N² 2, p. 27-43.

MARCH, H.; HERNÁNDEZ, M. y SAURÍ, D. Percepción de recursos convencionales y no convencionales en áreas sujetas a estrés hídrico: el caso de Alicante. Revista de Geografía Norte Grande, 2015a, No 60, p. 153-172.

MARCH, H.; HERNÁNDEZ, M. \& SAURÍ, D. Assessing domestic water use habits for more effective water awareness campaigns during drought periods: a case study in Alicante, eastern Spain. Natural Hazards and Earth System Sciences, 2015b, 15, p. 963-972. 
MARCH, H.; SAURI, D. \& RICO AMORÓS, A.M. The end of scarsity? Water desalinations the new cornucopia for the Mediterranean Spain. Journal of Hidrology, 2014, N519(C), p. 2642-2651.

MARTíNEZ GIL, F.J. La nueva cultura del agua en España. Bilbao: Bakeaz, 1997.

MAZÓN, A. y ALEDO, A. Los límites del turismo residencial: el caso de Torrevieja. Estudios Turísticos, 2005, No 165, p. 77-95.

MELOSI, M. The Sanitary City. Urban infrastructure in America from Colonial times to the present. Baltimore: Johns Hopkins University Press, 2000.

MORALES, A.; RICO, A.M. y HERNÁNDEZ, M. El trasvase Tajo-Segura. Observatorio medioambiental, 2005, No 8, p. 73-110.

MOROTE, A.F. Tipologías urbano-residenciales del litoral de Alicante: Repercusiones territoriales. Ciudad y Territorio. Estudios Territoriales, 2014, Vol. XLVI, Nº181, p. 431-443.

MOROTE, A.F. y HERNÁNDEZ, M. Jardines y urbanizaciones, nuevas naturalezas urbanas en el litoral de la provincia de Alicante. Documents d'Anàlisi Geogràfica, 2014, Vol. 60, N 3, p. 483-504.

OLCINA, J. y MOLTÓ, E. Recursos de agua no convencionales en España. Estado de la cuestión, Investigaciones Geográficas, 2010, № 51, p. 131-163.

PÉREZ, A.; GIL, E. y GÓMEZ, J.M. Las aguas residuales regeneradas como recurso para los regadíos de la demarcación hidrográfica del Segura (España). Boletín de la Asociación de Geógrafos Españoles, 2014, No 64, p. 151-175.

RICO, A.M. Plan Hidrológico Nacional y Programa A.G.U.A. Repercusión en las regiones de Murcia y Valencia, Investigaciones Geográficas, 2010, № 51, p. 235-267.

RICO, A.M. La mancomunidad de los canales del Taibilla: un modelo de aprovechamiento conjunto de fuentes convencionales y desalinización de agua marina. En: OLCINA, J. y RICO, A.M. (coordinadores). Libro jubilar en homenaje al profesor Antonio Gil Olcina. Alicante: Universidad de Alicante, 2014, p. 367-394.

RICO, A.M. y HERNÁNDEZ, M. Ordenación del territorio, escasez de recursos hídricos, competencia de usos e intensificación de las demandas urbano-turísticas en la Comunidad Valenciana. Documents d'Anàlisi Geográfica, 2008, № 51, p. 79-109.

RICO, A.M.; OLCINA, J.; PAÑOS, V. y BAÑOS, C. Depuración, desalación y reutilización de aguas en España (estudio regional). Barcelona: Oikos-Tau, 1998.

RICO, A.M.; OLCINA, J. \& SAURÍ, D. Tourist land use patterns and water demand: evidence from the Western Mediterranean. Land Use Policy, 2009, N²6, p. 493-501.

RICO, A.M.; SAURÍ, D.; OLCINA, J. \& VERA, J.F. Beyond megaproyects?. Water alternatives for Mass Tourism in coastal mediterranean Spain. Water Resour Manage, 2013, № 27, p. 553-565. 
RIDOLFI, E. Exploring the urban hydrosocial cycle in tourists environments. Investigaciones Geográficas, 2014, № 61, p. 17-38.

SAURI, D. \& DEL MORAL, L. Recent development in Spanish water policy. Alternatives and conflicts at the end of the hydraulic age. Geoforum, 2001, No 32, p. 351-362.

SCHMIDT, J.J. Historicising the hydrosocial cycle. Water Alternatives, 2014, Vol. 7, No 1, p. 220-234.

SEGUÍ, L.A. Sistemas de regeneración y reutilización de aguas residuales. Metodología para el análisis técnico-económico y casos. Barcelona: Tesis Doctoral, Universitat Politècnica de Catalunya. Departament d'Enginyeria Agroalimentària i Biotecnologia, 2004.

SWYNGEDOUW, E. Modernity and hybridity: Nature, regeneracionismo, and the production of the Spanish waterscape, 1890-1930. Annals of the Association of American Geographers, 1999, Vol. $89, N^{\circ} 3$, p. $443-465$.

SWYNGEDOUW, E. Social Power and the Urbanization of Water: Flows of Power. Oxford: Oxford University Press, 2004.

SWYNGEDOUW, E. Technonatural revolutions: The scalar politics of Franco's hydro-social dream for Spain, 1939-1975. Transactions of the Institute of British Geographers, 2007, Vol. 32, No 1, p. 9-28.

SWYNGEDOUW, E. The political economy and political ecology of the hidrosocial cycle. Journal of Contemporary Water Research and Education, 2009, Vol. 142, №1, p. 56-60.

SWYNGEDOUW, E. Into the Sea: Desalination as Hydro-Social Fix in Spain. Annals of the Association of American Geographers, 2013, Vol. 103, № 2, p. 261-270.

SWYNGEDOUW, E. Not a drop of water. State, modernity and the production of nature in Spain, 1898-2010. Environment and History, 2014, Vol. 20, № 1, p. 67-92.

SWYNGEDOUW, E. Liquid Power. Contested Hydro-Modernities in Twentieth Century Spain. Cambridge: MIT Press, 2015.

VERA, J.F. Turismo y Urbanización en el litoral alicantino. Alicante: Instituto de Estudios Juan Gil-Albert, 1987.

VERA, J.F. Transferencia de recursos y demandas turísticas. GIL OLCINA, A: y MORALES GIL, A. (Organizadores). Insuficiencias Hídricas y Plan Hidrológico Nacional. Alicante: CAM e Instituto Universitario de Geografía, Universidad de Alicante, 2002, p. 105-150. 